\title{
Design considerations for a dedicated gravity recovery satellite mission consisting of two pairs of satellites
}

\author{
D. N. Wiese • R. S. Nerem • F. G. Lemoine
}

Received: 24 February 2011 / Accepted: 3 June 2011 / Published online: 24 June 2011

(C) Springer-Verlag 2011

\begin{abstract}
Future satellite missions dedicated to measuring time-variable gravity will need to address the concern of temporal aliasing errors; i.e., errors due to high-frequency mass variations. These errors have been shown to be a limiting error source for future missions with improved sensors. One method of reducing them is to fly multiple satellite pairs, thus increasing the sampling frequency of the mission. While one could imagine a system architecture consisting of dozens of satellite pairs, this paper explores the more economically feasible option of optimizing the orbits of two pairs of satellites. While the search space for this problem is infinite by nature, steps have been made to reduce it via proper assumptions regarding some parameters and a large number of numerical simulations exploring appropriate ranges for other parameters. A search space originally consisting of 15 variables is reduced to two variables with the utmost impact on mission performance: the repeat period of both pairs of satellites (shown to be near-optimal when they are equal to each other), as well as the inclination of one of the satellite pairs (the other pair is assumed to be in a polar orbit). To arrive at this conclusion, we assume circular orbits, repeat groundtracks for both pairs of satellites, a 100-km inter-satellite separation distance, and a minimum allowable operational satellite altitude of $290 \mathrm{~km}$ based on a projected 10-year mission

D. N. Wiese $(\varangle) \cdot$ R. S. Nerem

Colorado Center for Astrodynamics Research,

University of Colorado at Boulder, $431 \mathrm{UCB}$, Boulder, CO 80309, USA

e-mail:wiese@colorado.edu

R. S. Nerem

e-mail: nerem@colorado.edu

F. G. Lemoine

NASA Goddard Space Flight Center, Planetary Geodynamics Laboratory, Greenbelt, MD 20771, USA

e-mail: frank.g.lemoine@ nasa.gov
\end{abstract}

lifetime. Given the scientific objectives of determining timevariable hydrology, ice mass variations, and ocean bottom pressure signals with higher spatial resolution, we find that an optimal architecture consists of a polar pair of satellites coupled with a pair inclined at $72^{\circ}$, both in 13 -day repeating orbits. This architecture provides a $67 \%$ reduction in error over one pair of satellites, in addition to reducing the longitudinal striping to such a level that minimal post-processing is required, permitting a substantial increase in the spatial resolution of the gravity field products. It should be emphasized that given different sets of scientific objectives for the mission, or a different minimum allowable satellite altitude, different architectures might be selected.

Keywords Time variable gravity - GRACE - Temporal aliasing errors · Constellations · Satellite geodesy

\section{Introduction}

The Gravity Recovery and Climate Experiment (GRACE) satellite mission has demonstrated the ability to measure mass variations on the Earth at monthly to sub-monthly resolution (Tapley et al. 2004a; Bruinsma et al. 2010). There is tremendous potential in the science applications of this data set, including quantifying variations in continental hydrology, measuring ocean currents and ocean bottom pressure variations, and sensing the loss and accumulation of ice in the polar regions as well as glaciated regions around the globe. Because GRACE senses these signals at large spatial scales ( $\sim 400 \mathrm{~km}$ ), mass variations in smaller drainage basins, for example, cannot be quantified by GRACE. Thus, it is of primary interest for future missions to have increased spatial resolution in the time-variable gravity field products. One 
potential benefit of this data set would be to aid in regional water management practices.

In designing future missions, we have now reached a point where the spatial resolution will no longer be limited by sensor errors, but rather by temporal aliasing errors (Wiese et al. 2011; Loomis 2009; Visser et al. 2010), assuming that future missions will use a laser interferometer for the intersatellite ranging (Pierce et al. 2008; Bender et al. 2003), and the spacecraft will fly drag-free at lower altitudes, similar to the Gravity field and steady-state Ocean Circulation Explorer (GOCE) (Drinkwater et al. 2007) mission. Temporal aliasing errors are defined as errors due to temporal undersampling of geophysical signals of interest (e.g., continental hydrology) as well as mis-modeling unwanted high-frequency mass variations (e.g., atmosphere and ocean tides).

We aim to directly reduce the effect of temporal aliasing errors by adding a second pair of satellites, thus increasing the sampling frequency of the mission and improving the spatial resolution of the derived gravity field products. In theory, if enough pairs of satellites were placed in proper orbits, one could sample the gravity field at a high enough frequency such that temporal aliasing errors would be largely eliminated. However, there could still be a problem of signal separation given this scenario. Having a dozen, if not more, satellite pairs to accomplish such a feat is cost-prohibitive at this point. As such, this paper focuses on the more interesting question of optimizing the orbits of two pairs of satellites.

One reason that the GRACE gravity field products have limited spatial resolution is the fact that the polar orbiting two-satellite collinear architecture has little East-West sensitivity to the gravity field. This leads to a strong correlation between coefficients of a fixed order and the same parity of degree, manifesting as longitudinal stripes in the gravity solutions. One common method used to remove the stripes is to apply a decorrelation filter (Swenson and Wahr 2006) to the data (known as 'destriping'). The gravity fields are also typically smoothed with a Gaussian filter (Wahr and Molenaar 1998) to average through any remaining errors. The resulting product is a map of global mass variations at large spatial scales. While substantially reducing the level of errors in the gravity solutions, these two processes have the undesired effects of removing real geophysical signal as well as reducing the spatial resolution of the solutions, particularly in regions with high signal to error ratios. Hence, it would be advantageous if future missions did not have to rely on these techniques.

While simulation studies have shown that alternate formations, such as cartwheels and pendulums, reduce the level of striping in the gravity solutions (Elsaka 2010; Wiese et al. 2009), these types of formations are much more difficult to implement from an engineering standpoint. Alternately, Bender et al. (2008) suggested that having two pairs of collinear satellites, one pair in a polar orbit and one in a lower inclined orbit, would improve the East-West sensitivity to the gravity field variations, thus leading to a reduction in the level of striping. This concept is explored in this paper.

\section{Orbit design considerations}

The goal of this paper is to optimize the orbits of two pairs of collinear satellites for improving the spatial resolution of the derived gravity field products for future missions over what GRACE provides. The search space for such a problem is extremely large, and is further complicated when considering that the selected orbits will be a strong function of the science goals of the mission. For instance, if the primary goal of the mission is to determine continental hydrology (excluding ice) at small spatial scales, then one might place the satellites in orbits with dense coverage over these regions, but less coverage over the polar regions. This would most likely result in decreased sensitivity to determining ice mass variations in Greenland and Antarctica. However, if the primary science objective is to determine ice mass variations in Greenland, then a different mission architecture would be selected. This study assumes that the science goals of the mission are to determine continental hydrology, ice mass variations, and ocean bottom pressure signals over the entire globe, with each area of science being weighted equally.

Considering strictly the satellite orbits, one can characterize the mission performance, $P$, given two pairs of collinear satellites, via the following:

$P=f\left(X_{1}, X_{2}, \Delta v_{1}, \Delta v_{2}, L\right)$.

In Eq. 1, $X_{1}$ and $X_{2}$ are the state (position and velocity) of the lead spacecraft of the first and second pair of satellites, respectively, $\Delta v_{1}$ and $\Delta v_{2}$ are the separation distances between the first and second pairs of satellites, respectively, and $L$ is the amount of time that data are collected to form a single solution. It is most convenient to represent the state of the spacecraft in terms of mean Keplerian orbital elements as

$X_{1}=f\left(a_{1}, e_{1}, i_{1}, \Omega_{1}, \omega_{1}, v_{1}\right)$

$X_{2}=f\left(a_{2}, e_{2}, i_{2}, \Omega_{2}, \omega_{2}, v_{2}\right)$

Here, $a$ is the semimajor axis, $e$ is the eccentricity, $i$ is the inclination, $\Omega$ is the longitude of ascending node, $\omega$ is the argument of perigee, and $v$ is the true anomaly. Coupling Eqs. 1 and 2, one can see that the mission performance of this type of architecture will be directly related to 15 parameters. Adding additional satellite pairs will increase the number of variables by seven for each pair of satellites added. It is desirable to reduce the number of independent variables and narrow down the search space by making appropriate assumptions. 
First, the inter-satellite separation distances, defined as $\Delta v_{1}$ and $\Delta v_{2}$, will likely be chosen based on the satellite-tosatellite ranging instrument requirements. Future missions are likely to use a laser interferometer, for which a $100-\mathrm{km}$ separation distance is chosen as a trade-off between instrument performance as well as relative accuracy in determining short wavelength and long wavelength features in the gravity field (Wiese et al. 2009). Fixing this distance allows us to eliminate two of the variables, $\Delta v_{1}$ and $\Delta v_{2}$, from the search space.

Next, it can be assumed that the spacecraft should fly in circular orbits to minimize any relative changes in distance due to having eccentric orbits, as GRACE does. Fixing the eccentricity to zero eliminates two additional parameters.

Given circular orbits, the argument of perigee, $\omega$, becomes ill-defined. Hence, we can now define the argument of latitude, $u$, which is equal to the sum of the true anomaly and the argument of perigee $(u=\omega+v)$. The argument of latitude defines the position of the satellite in its orbit about the Earth with respect to $\Omega$. While this is a parameter that could have an effect on the gravity solution, it is impossible to determine what the optimal satellite position should be due to the extremely complex nature of the problem. For example, it would be optimal if, during a flooding event, the satellite flew over the region of interest. However, it is impossible to know when this event might occur in the future, making it very difficult to optimize.

While optimizing $u_{1}$ and $u_{2}$ independently is not feasible, one could think of optimizing the relative difference in the argument of latitude between the two pairs of satellites in an effort to meet certain temporal groundtrack crossing constraints (i.e., the second satellite pair will fly over a location on the Earth a specified amount of time after the first satellite pair flew over the same location). The same argument holds for the longitude of ascending node, $\Omega$, in a spatial sense. That is, in an absolute sense it is impossible to determine what the optimal values for $\Omega_{1}$ and $\Omega_{2}$ should be, since we cannot predict the time and location of mass variations on the Earth years in advance. However, the relative difference between the ascending nodes of the two pairs of spacecraft could be optimized to provide a required spatial constraint on the combined groundtrack pattern of the two satellite pairs. Thus, $\Omega_{1}$ and $\Omega_{2}$, along with $u_{1}$ and $u_{2}$ can be reduced to two new parameters: $\Delta \Omega_{12}$, and $\Delta u_{12}$. The first, $\Delta \Omega_{12}$, provides a spatial constraint on the groundtrack pattern of the two satellite pairs while the second, $\Delta u_{12}$ provides a temporal constraint on the groundtrack pattern. It is expected that $\Delta u_{12}$ can only be optimized if the periods of both satellites pairs are equal to each other, which would require that $a_{1}=a_{2}$. Otherwise, there will be a secular drift rate in the time that the two pairs of satellites cross the same location on the Earth which cannot be controlled.
Next, we can consider the inclination of the satellites. In order to provide global coverage of the Earth, at least one of the pairs of satellites must be in a near-polar orbit. Thus, this can be set as a constraint. The inclination of the second pair of satellites, however, is free to vary. The problem has now been reduced from one with 15 parameters to one with only six, and can be represented via the following:

$P=f\left(a_{1}, a_{2}, i_{2}, \Delta u_{12}, \Delta \Omega_{12}, L\right)$.

Let us now discuss the semimajor axis of the two pairs of satellites. GRACE was launched into an altitude of approximately $490 \mathrm{~km}$ (Tapley et al. 2004b) in which the altitude of the satellites continuously decays primarily due to atmospheric drag forces. Currently, GRACE is at a nominal measurement altitude of $460 \mathrm{~km}$ (Center for Space Research 2011). GOCE, alternatively, which is designed to measure the static gravity field of the Earth to high spatial resolution, flies at a much lower altitude than GRACE, at approximately $255 \mathrm{~km}$. It employs a single-axis drag-free system where non-conservative forces are measured in real time and are compensated for using thrusters to maintain a nominal altitude. GOCE was designed for a lifetime of 2 years and is limited by the amount of propellant onboard to keep the spacecraft at the nominal measurement altitude. This can be contrasted with the GRACE mission, which is currently in its ninth year of operations. It should be noted, however, that due to the extended solar minimum at the end of the past decade, GOCE is now expected to continue performing several years beyond its mission lifetime (Fehringer et al. 2010).

It is envisioned that future GRACE-type missions will also employ drag-free technology, allowing one to fly at a lower altitude with increased sensitivity to short wavelength features in the gravity field. With this in mind, one can set a minimum bound on the altitude of the spacecraft which depends on many factors, including, but not limited to the design lifetime of the satellites, the amount of propellant available, the type of thrusters used, the cross-sectional area of the spacecraft, and the magnitude of the atmospheric density. Some work has been done to this end, by Marchetti et al. (2008) and St. Rock et al. (2006), examining the performance of drag-free control systems in low-Earth orbit, and the mission lifetimes associated with various thrusters. Figure 1 depicts the results from each respective paper, along with the initial estimate for the GOCE mission, assuming the same initial mass propellant fraction as the GRACE mission (0.18). Note that the results from St. Rock et al. (2006) have been scaled down by a factor of two to account for variable specific impulse and control system use that was not considered in the analysis.

Given that GRACE is quickly approaching its tenth year of operations, it seems both reasonable and desirable to design future missions with a lifetime of at least 10 years. Figure 1 illustrates that a $290-\mathrm{km}$ altitude allows the satellites 


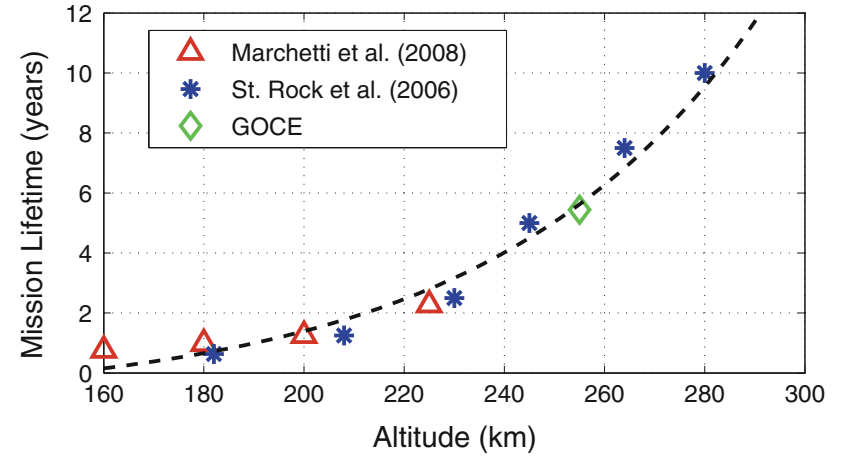

Fig. 1 Mission lifetime as a function of altitude assuming an initial mass propellant fraction of 0.18

to remain in orbit for 10 years; thus, this was selected as the minimum altitude for this study. Note that this calculation is very approximate, and a rigorous analysis of a control system in the appropriate environment would need to be made to refine the targeted altitude; however, it is valid as a first-order approximation and sufficient for the purposes of this study.

The last parameter which needs to be discussed from Eq. 3 is $L$, the length of time that data are collected. The parameter $L$ will depend primarily on the targeted spatial and temporal resolution of the mission. In principle, the product of the spatial resolution and temporal resolution of a mission is constant; that is, given a fixed number of satellite pairs, one cannot improve the spatial resolution without sacrificing temporal resolution and vice-versa (Visser et al. 2010). Given homogeneous groundtrack spacing, the spatial resolution of a mission can be approximated by the Colombo-Nyquist rule, which states the maximum resolvable degree of the gravity field is equal to half of the number of orbital revolutions of the satellites (Colombo 1984), guaranteeing gravity solutions that are homogeneous in longitude (Visser et al. 2011). While larger values of $L$ theoretically lead to better spatial resolution, they also allow for greater accumulation of temporal aliasing errors. Varying the $L$ parameter should lend insight into proper trade-offs between increasing the spatial resolution of the solutions and mitigating the effect of temporal aliasing errors.

There is one more point to be mentioned concerning $L$. One drawback of GRACE is the lack of an altitude control system. This leads to variability in the groundtrack pattern of GRACE, and subsequent variability in the quality of the monthly solutions. This was discussed in Klokočník et al. (2008) and Wagner et al. (2006), showing the degradation in gravity solutions from GRACE in the fall of 2004 when the satellites passed through a 61/4 resonance orbit. Due to the success of the GOCE mission implementing a drag-free system and maintaining a constant orbital altitude, it seems advantageous to consider only repeat groundtracks for future missions. Imposing this constraint assures consistent quality in the time-variable gravity solutions. It should be noted, however, that maintaining repeat groundtracks with a pair of satellites will be more demanding than for a single satellite, like GOCE. Tolerances on the formation dynamics will be set based on instrument performance criteria and how tightly one needs to maintain the repeat groundtrack (presumably this will be a function of the selected orbit and what other repeat orbits exist close to the operational altitude). These parameters will need to be given careful consideration should such a mission be flown.

From Kaula (1966), even zonal coefficients in addition to a nonlinear $\left(J_{2}\right)^{2}$ contribute to the secular rate of the node, $\dot{\Omega}$. However, all terms are second order when compared with the contribution of $J_{2}$; thus, we design the repeat groundtracks by considering only this term. Given a desired eccentricity and inclination, there are only certain values for the semimajor axis which will satisfy the conditions for a repeating groundtrack. One can obtain the appropriate values for semimajor axis, $a$, by solving the following equation (Vallado 2001):

$C_{2} a^{7 / 2}+C_{1} a^{2}+C_{0}=0$,

where

$C_{2}=\frac{l}{k} \omega_{\mathrm{e}}$

$C_{1}=-\sqrt{\mu}$

$C_{0}=\frac{\alpha}{4 \epsilon^{4}}\left[\frac{l}{k} 2 \cos i+1-5 \cos ^{2} i-\left(3 \cos ^{2} i-1\right) \epsilon\right]$,

in which

$\alpha=3 \sqrt{\mu} J_{2} R_{\mathrm{e}}^{2}$

$\epsilon=\left(1-e^{2}\right)^{\frac{1}{2}}$.

In these sets of equations, $\mu$ is the gravitational constant of the Earth, $J_{2}$ is the negative of the unnormalized $C_{20}$ coefficient describing the oblateness of the Earth, $R_{\mathrm{e}}$ is the radius of the Earth, $\omega_{\mathrm{e}}$ is the rotation rate of the Earth, $k$ is the desired number of nodal days it takes for the satellites to repeat, and $l$ is the number of orbital revolutions the satellites perform in $k$ nodal days. It should be noted that $k / l$ must be irreducible, and $l$ is given by

$l=\frac{k(\dot{\omega}+\dot{M})}{\left(\omega_{\mathrm{e}}-\dot{\Omega}\right)}$.

In Eq. 7, $\dot{\omega}$ and $\dot{M}$ are the secular drift rates of the argument of perigee and mean anomaly, respectively, due to the oblateness of the Earth.

Thus, selecting a particular value for $L$ inadvertently imposes an additional constraint on either $a_{1}$ or $a_{2}$ : that the value for $a$ must put the satellite in a repeat orbit. It is not imperative that both satellite pairs have a value of $k$ equal to that of $L$, but one pair must. It has been pointed out by Bender et al. (2008) that perhaps the most effective way to design the 
Table 1 Constraints imposed on design criteria to reduce the search space for an optimal architecture

\begin{tabular}{ll}
\hline Parameter & Constraint \\
\hline$a_{1}, a_{2}$ & $290 \mathrm{~km}$ minimum; repeat groundtrack \\
$e_{1}, e_{2}$ & 0 (circular orbits) \\
$i_{1}$ & $90^{\circ}$ \\
$\Delta \nu_{1}, \Delta \nu_{2}$ & $100 \mathrm{~km}$ \\
$L$ & $L=k_{2} ; k_{1} \leq k_{2}$ \\
\hline
\end{tabular}

architecture would be to have a lower inclined pair in a longer repeat period (RP) coupled with a polar pair of satellites in a shorter RP. This would lead to more homogeneous spacing in the combined groundtrack pattern of the two pairs of satellites, since, by nature, groundtracks are more dense over the poles than the equator. In this scenario, the lower inclined pair would be selected to have a value of $k$ equal to $L$, while the RP of the polar pair of satellites would be allowed to vary, but would be constrained to be less than or equal to that of the lower inclined pair. Thus, all such combinations should be explored.

Finally, taking into consideration the above discussion, Eq. 3 can be rewritten as

$P=f\left(k_{1}, k_{2}, i_{2}, \Delta u_{12}, \Delta \Omega_{12}\right)$.

In Eq. $8, k_{1}$ is the RP of the polar pair of satellites and $k_{2}$ is the RP of the other pair of satellites for which the inclination can vary. Note that the additional constraints which are imposed are that $k_{2}=L$ and that $k_{1} \leq k_{2}$. One additional caveat that should be mentioned is that $k$ is expressed in units of nodal days, while $L$ is typically expressed in units of solar days, since the data processing is usually set up to handle daily batches of data. This means that typically a solution will have slightly more data (a few hours) than what is taken during the full repeat period of the satellites. Table 1 is a list of all constraints that were imposed to arrive at Eq. 8 .

Using the constraints listed in Table 1, Eq. 8 has been reduced from one that initially was a function of 15 variables to one that is now a function of only five variables. Furthermore, it is expected that the values selected for $k_{1}, k_{2}$, and $i_{2}$ will have the most influence on how well the mission performs. $\Delta u_{12}$ and $\Delta \Omega_{12}$ are expected to have much smaller impacts.

It should further be stressed that this type of analysis is considerably biased towards the minimum altitude chosen, in this case, $290 \mathrm{~km}$. To illustrate this, Fig. 2 shows the closest altitude to $290 \mathrm{~km}$ (without going below it) for different values of $k_{1}$ for a polar pair of satellites.

Figure 2 illustrates how results could be biased towards the minimum allowable altitude. For example, the closest 8-day RP groundtrack to $290 \mathrm{~km}$ exists at an altitude of $291 \mathrm{~km}$, versus $374 \mathrm{~km}$ for a 12-day RP. The lower altitude given by the

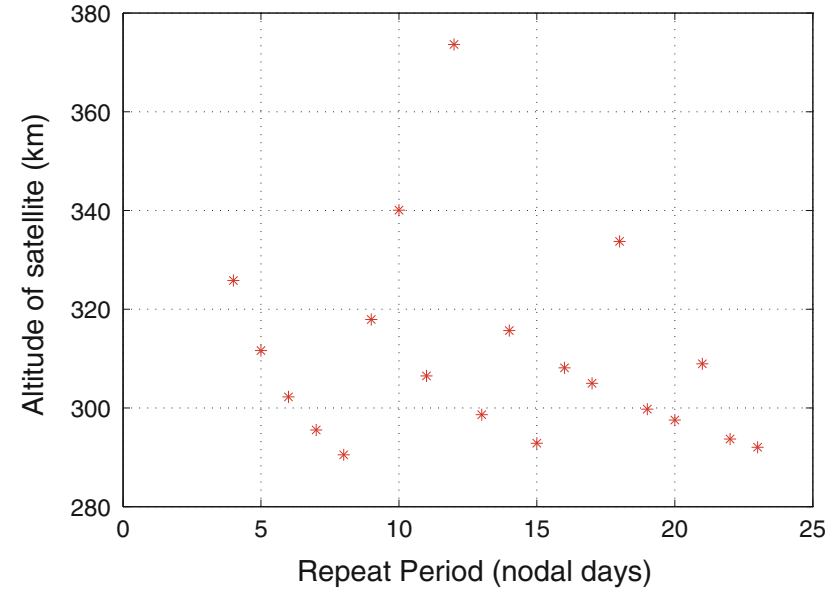

Fig. 2 Necessary altitude to maintain specific repeat periods for a polar pair of satellites

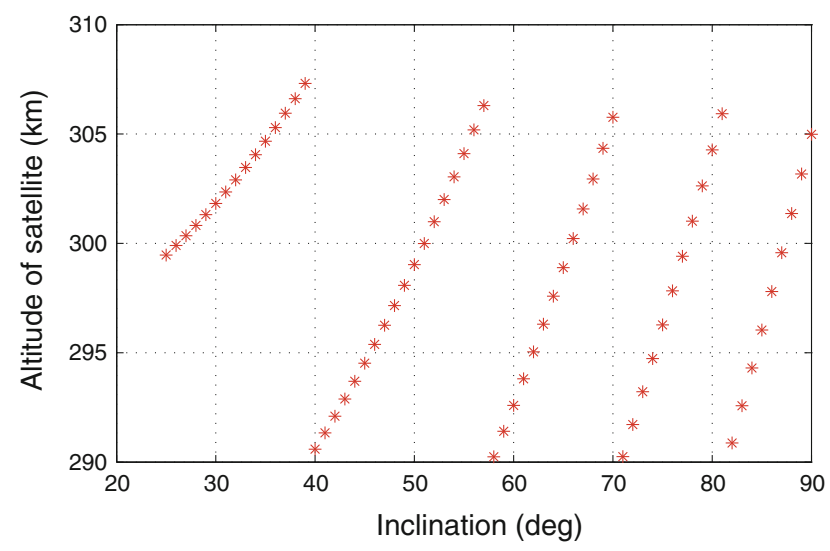

Fig. 3 Necessary altitude to maintain a 17-day repeat period at different inclinations

8-day RP orbit could trump any benefit that collecting data for 12 days versus 8 days might add.

The same analysis can be done if one fixes a value for $k_{2}$, but lets $i_{2}$ vary. Figure 3 shows how the altitude necessary to maintain a 17-day RP orbit changes as a function of inclination.

Hypothetically speaking, if a $70^{\circ}$ inclination were an optimal value for $i_{2}$ speaking strictly in terms of inclination, Fig. 3 suggests that this study might find that a $71^{\circ}$ inclination is the optimal value for $i_{2}$ since this orbit is $16 \mathrm{~km}$ lower in altitude than the orbit with a $70^{\circ}$ inclination. This analysis shows how the altitude, repeat period, and inclination are inherently coupled together, and an optimal set of orbital parameters will be a strong function of the mission constraints.

\section{Methodology}

Numerical simulations were used to explore the full search space of parameters for the investigation. This was deemed 
necessary due to the variable spatio-temporal sampling characteristics of different mission architectures along with the unpredictable nature of geophysical signals and temporal aliasing errors. The primary parameters of interest in Eq. 8 are $k_{1}, k_{2}$, and $i_{2}$. The following are the ranges of values explored for these parameters:

$k_{1}, k_{2}=[4,23]$ days; $k_{1} \leq k_{2}$

$i_{2}=\left[25^{\circ}, 90^{\circ}\right]$

Using a step size of $5^{\circ}$ for $i_{2}$, it is readily seen that 2,940 simulations were run to explore the entire search space of the $k_{1}, k_{2}$, and $i_{2}$ variables. After the initial results narrowed down a more appropriate range of values for $i_{2}$, a $1^{\circ}$ step size was used. While a more systematic method could have been implemented to explore the search space and reduce computation time, this would have led to greater uncertainty in the validity of our analysis due to the variable spatiotemporal characteristics of geophysical signals, temporal aliasing errors, and the sampling behavior of the different architectures.

\subsection{Simulation procedure}

The GEODYN software package (Pavlis et al. 2010) (an orbit determination program) coupled with SOLVE (Ullman 1997) (a linear systems solver), both from NASA Goddard Space Flight Center, was used to perform the numerical simulations. In an effort to create as realistic of a simulation environment as possible, we model the dominant mass variations in the Earth system, including the atmosphere, oceans (mass redistribution due to atmospheric forcing), tides (ocean, atmosphere, and solid Earth), continental hydrology, and ice sheets. Table 2 shows the simulation design. Throughout the paper, when we refer to recovering hydrology signals, we actually mean recovering continental hydrology, while recovering ice signals refers to recovering ice mass variations in Greenland and Antarctica.

The static gravity field model used, EIGEN-GL04C, includes data from the GRACE and LAGEOS missions along with surface gravity data from altimetry over the oceans and gravimetry over the continents (Förste et al. 2008). The

Table 2 Simulation and model definitions

\begin{tabular}{lll}
\hline Models & Truth & Nominal \\
\hline Static gravity field & EIGEN-GL04C & EIGEN-GL04C \\
Ocean tide model & FES2004 & GOT00 \\
Atmospheric model & ECMWF & NCEP \\
Ocean model & OMCT & MOG-2D \\
Hydrological model & GLDAS & None \\
Ice model & ESA & None \\
\hline
\end{tabular}

atmospheric models used are 3-h ECMWF surface pressure fields (Klinker et al. 2000) and 6-h NCEP Reanalysis fields (Kalnay et al. 1996) while the ocean models are the baroclinic OMCT model which is used as a dealiasing product for GRACE (Flechtner 2007), along with the barotropic MOG2D model, both of which are forced by ECMWF surface pressure (Carrère and Lyard 2003). The tide models used are of two generations. GOT00.2 uses almost 8 years of TOPEX and Poseidon data, supplemented in shallow seas and in polar seas (latitudes above $66^{\circ}$ ) by ERS- 1 and ERS-2 data and uses FES94 as an apriori model (Ray 1999). FES2004 is a hydrodynamic model that not only assimilates more recent altimetry data, but also includes that from TOPEX (Lyard et al. 2006). The 3-h GLDAS/Noah land-surface model is used to describe continental hydrology (Rodell et al. 2004) and the 6-h ESA ice model describes the ice sheet dynamics in Greenland and Antarctica, and was provided by the European Space Agency (ESA) (van Dam et al. 2008). All simulations are arbitrarily run for January of 2003. The ice model is defined from 1995 to 2006 with 1995 being the reference time; as such, the magnitude of the ice signal in 2003 is significant. All models are represented to spherical harmonic degree and order 100 in the simulations with the exception of the NCEP and MOG-2D models, which are represented to spherical harmonic degree and order 72 . Note that solid Earth and atmospheric tides are included in the simulation, but errors are not considered.

Table 2 shows that this particular simulation is designed to recover hydrology and ice mass variations in the presence of model errors from ocean tides and atmospheric and oceanic mass variations, defined as the differences between the two sets of models. One can also calculate ocean bottom pressure variations from the above simulation by treating the NCEP and MOG-2D models as forward models and examining differences with respect to the truth models over the oceans. All models are interpolated linearly in time during the simulation process.

The spacecraft are assumed to be equipped with a laser interferometer as a replacement for the microwave ranging instrument onboard the GRACE mission. The noise assumed for the laser interferometer is $5 \mathrm{~nm} / \sqrt{\mathrm{Hz}}$ (Young et al. 1999; Mueller et al. 2005; Wiese et al. 2009). Additionally, the spacecraft are assumed to fly drag-free, such that they maintain their nominal orbital altitudes. The noise on the dragfree system is taken to be $0.01 \mathrm{~nm} / \mathrm{s}^{2} / \sqrt{\mathrm{Hz}}$, which is similar to that of the GOCE mission. Spacecraft position errors are added as white noise with a 1-cm RMS in the radial, alongtrack, and crosstrack directions. Simulations show that with these levels of error, temporal aliasing errors from mismodeling atmosphere and ocean signals dominate the error budget by more than two orders of magnitude over the level of error from the drag-free system and laser interferometer (Wiese et al. 2011). Thus, any improvements that certain 
architectures offer over other architectures will be attributed to reducing temporal aliasing errors.

The simulations are carried out in two steps. First, the selected satellite orbits are integrated through both the truth and nominal sets of force models independently. Noise on the laser interferometer, drag-free system, and satellite positions are added to the truth set of measurements. The residuals between the inter-satellite range-rate measurements coupled with the satellite position measurements are then used to estimate a correction to the spacecraft state. This step is performed to account for a change in energy of the system due to a different set of force models. The second step involves integrating the spacecraft orbits through the nominal set of force models using the updated spacecraft state. The range-rate residuals are then used exclusively to estimate any additional corrections to the state of the spacecraft along with corrections to the geopotential coefficients. Spacecraft position measurements are not used in this step, as the spacecraft states are converted to baseline elements via Rowlands et al. (2002), and nine out of the twelve parameters are constrained during the estimation process. Data are collected in one-day batches and SOLVE is used to combine $k_{2}$ days of data together to arrive at the final multi-day solution.

The computation time associated with such a large matrix of simulations is expensive, and increasing the degree of estimation exponentially increases the processing time. As such, a subset of simulations was carried out to both degree 60 and degree 100 to ensure consistency between the two, in hopes of being able to run the matrix of simulations to degree 60 . It was expected that the two would correlate; however, the results were surprising, showing smaller correlations than expected. This is discussed in detail in Sect. 4.1. As a result, all simulations were run to degree and order 100.

\subsection{Performance metrics}

There are several ways one can quantify the performance of a gravity recovery satellite mission, given by $P$ in Eq. 8. As discussed in Sect. 2, $P$ depends substantially on what the scientific goals of the mission are. For this study, we took the liberty of defining the scientific goals of the mission to be increasing the spatial resolution of the recovered hydrology, ice, and ocean bottom pressure signals over what GRACE provides. Each area of science is weighted equally; hence, one hopes to minimize the error, $E$, given by

$E=\frac{E(\mathrm{H})+E(\mathrm{I})+E(\mathrm{O})}{3}$

In Eq. 11, $E(\mathrm{H})$ represents the error in determining hydrology, $E(\mathrm{I})$ is the error in determining ice mass variations, and $E(\mathrm{O})$ is the error in determining ocean bottom pressure signals. There are many methods and tools which one can use to analyze error and quantify $E(\mathrm{H}), E(\mathrm{I})$, and $E(\mathrm{O})$ on both global and regional scales. It was pointed out in Han and Ditmar (2008) that global error metrics lack sophistication as they disregard the different spatial distributions of signals and errors, making it desirable to analyze errors on a regional basis.

There are several types of localized analysis one can use, including, but not limited to, localized averaging kernels (Swenson and Wahr 2002) and a spatiospectral localization technique (Han and Simons 2008; Han and Ditmar 2008; Simons et al. 2006; Wieczorek and Simons 2005). While these are effective techniques to analyze regional mass variations, a complete analysis of this kind requires one to select multiple regions of varying size, signal strength, error characteristics, etc., to properly analyze the results. Since this study is already computationally expensive, it is desirable to use a global metric to quantify $E$ to narrow down the search space and identify a select few mission architectures for further analysis on a regional scale. This type of regional analysis will be addressed in future work.

In a global sense, one of the most common ways to look at error is using degree variances. While this is a valuable tool to examine errors in the spectral domain globally, the end user of the GRACE data is more interested in what is happening in the spatial domain. Thus, it was determined that the metric used for calculating $E(\mathrm{H}), E(\mathrm{I})$, and $E(\mathrm{O})$ would be differencing the truth signals and the recovered signals to obtain a spatial plot of errors. The spatial plot of errors is represented on a $1^{\circ} \times 1^{\circ}$ grid, and an area-weighted RMS of the errors for each signal is calculated and substituted into Eq. 11 for $E(\mathrm{H}), E(\mathrm{I})$, and $E(\mathrm{O})$. While this is not a perfect representation for the performance of a mission by itself, it does give a very good indication of how changing $k_{1}, k_{2}$, and $i_{2}$ affects the ability of the satellites to recover the geophysical signals that we are interested in.

Figure 4 illustrates how $E$ is calculated, showing the truth signals (left), recovered signals (middle), and the recovered signals after being destriped via Swenson and Wahr (2006) and smoothed with a $300-\mathrm{km}$ Gaussian averaging radius (right), for recovering both hydrology and ice mass variations (top), as well as ocean bottom pressure signals (bottom). This simulation is for a single pair of polar orbiting satellites in a 13-day RP at $299 \mathrm{~km}$. The figures have been truncated at degree 60 and are expressed in $\mathrm{cm}$ of equivalent water height $(\mathrm{EWH})$. Note that in this case, the truth signal for hydrology and ice is defined as the 13-day average of GLDAS + ESA, while the truth ocean signal is defined as the 13-day average of ECMWF + OMCT. Any non-zero mean between the atmosphere and ocean models over the time span of interest is compensated for in the post-processing when estimating hydrology and ice signals.

When calculating $E$, one uses a spatial plot of the errors which is obtained by differencing the truth signals, given by the left set of figures, with the recovered signals, given by the 

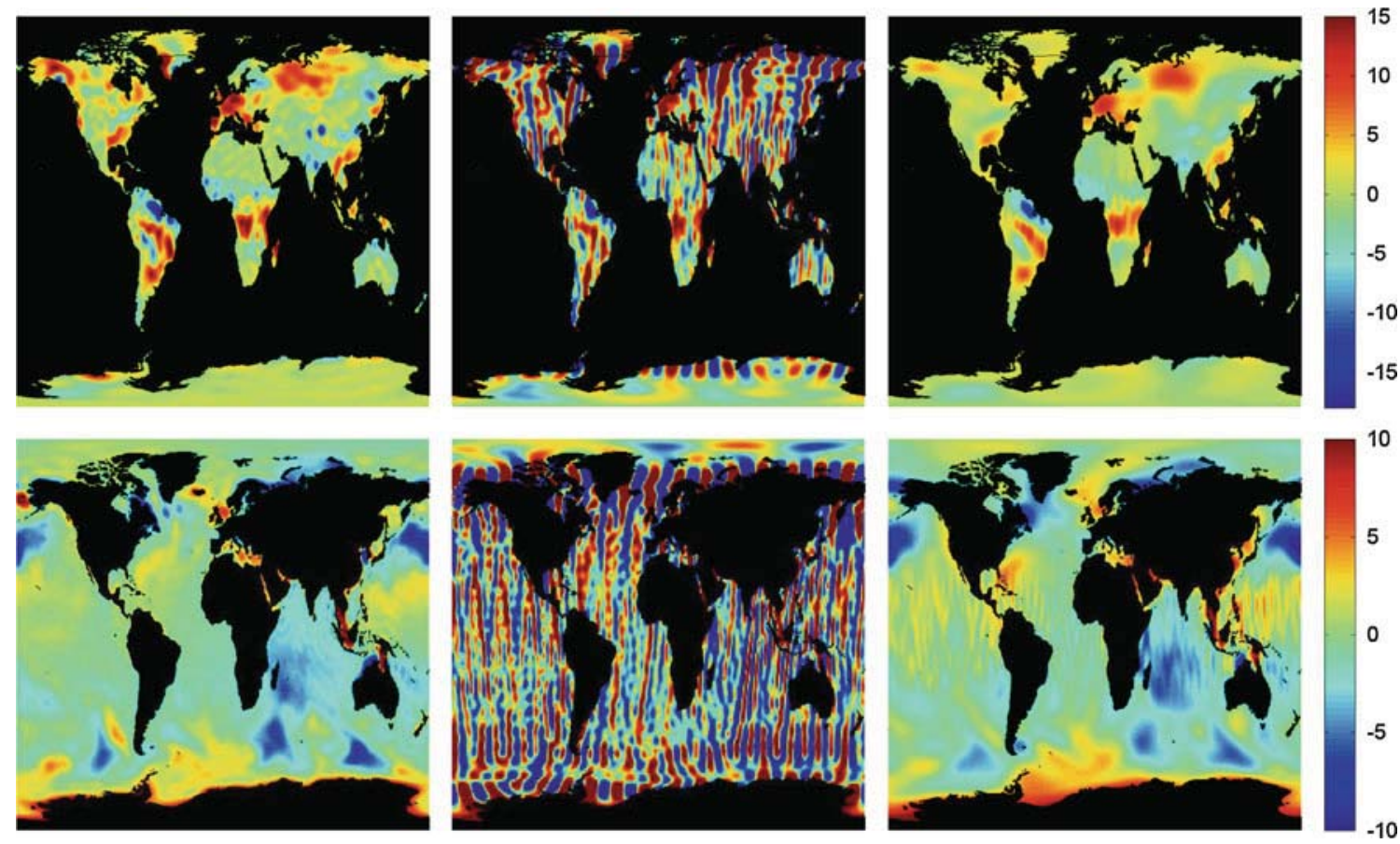

Fig. 4 Truth signals (left), recovered signals (middle), and recovered signals after PP (right) for recovering hydrology and ice mass variations (top row) and ocean bottom pressure signals (bottom row) given a polar

Table 3 Signal and error associated with Fig. 4

\begin{tabular}{llll}
\hline & Signal $(\mathrm{cm})$ & Error $(\mathrm{cm})$ & \\
\cline { 3 - 4 } & & No PP & PP \\
\hline Hydrology & 4.67 & 13.97 & 2.47 \\
Ice & 2.12 & 7.54 & 2.06 \\
Ocean & 2.46 & 11.04 & 1.71 \\
Average & $S=3.08$ & $E=10.85$ & $E=2.08$ \\
\hline
\end{tabular}

Units are expressed in $\mathrm{cm}$ of EWH

middle and right sets of figures. Furthermore, one can calculate the power in the truth signals, $S$, for hydrology $(S(\mathrm{H}))$, ice mass variations $(S(\mathrm{I}))$, and ocean bottom pressure $(S(\mathrm{O}))$ in the same manner that the error is calculated in Eq. 11. Table 3 illustrates the signal and error associated with Fig. 4 in $\mathrm{cm}$ of EWH for the recovered signals with no post-processing (PP) and the recovered signals after PP. In this case, it is seen that the error exceeds that of the signal with no PP applied, but after destriping and smoothing the solutions the level of error is reduced to be lower than the signal.

Note that throughout the paper, $E$ is obtained by taking the solutions to degree 100 and truncating them at degree 60 to make the spatial maps. The reason for doing this is that it was found that if the results are truncated at degree 60 , des- pair of satellites at $299 \mathrm{~km}$ in a 13-day repeating groundtrack. Units are in $\mathrm{cm}$ of equivalent water height

triping at lower latitudes is generally not required, resulting in a gravity solution with better spatial resolution.

\section{Results}

This section shows the most important results from the numerical simulations in an effort to optimize $k_{1}, k_{2}$, and $i_{2}$. Additionally, Sect. 4.5 discusses groundtrack patterns obtained by tuning $\Delta \Omega_{12}$ and $\Delta u_{12}$. This section begins with a discussion on the impact of performing simulations to degree 60 versus degree 100 .

\subsection{Degree of estimation}

It was found that the calculated error, $E$, varies substantially for certain cases depending on if the simulations are run to degree and order 60 or 100. To explain this, an example case is shown. When simulations are carried out to degree and order 60, one of the better performing mission architectures consists of a polar pair of satellites in an 8-day RP at an altitude of $291 \mathrm{~km}$ coupled with a lower inclined pair $\left(72^{\circ}\right)$ in a 13-day RP at an altitude of $290 \mathrm{~km}$. The error, $E$, from Eq. 11, is calculated to be $4.64 \mathrm{~cm}$ EWH for this case. Conversely, when the simulations are extended to degree and order 100 , but truncated at degree 60 for a fair comparison, 
Fig. 5 Logarithm of the error in the coefficients for a simulation carried out to degree 60 (left) and a simulation carried out to degree 100 (right)

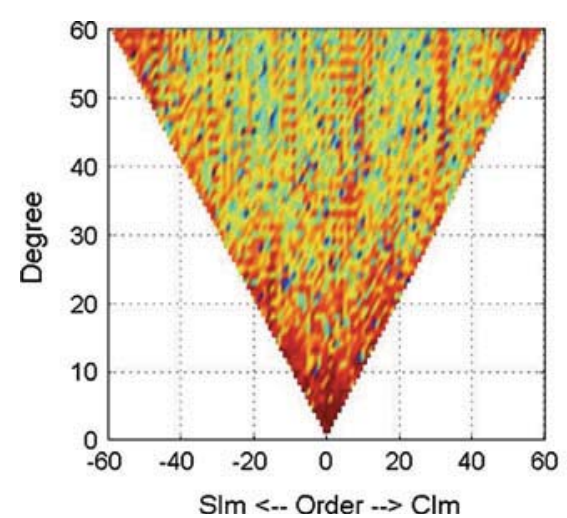

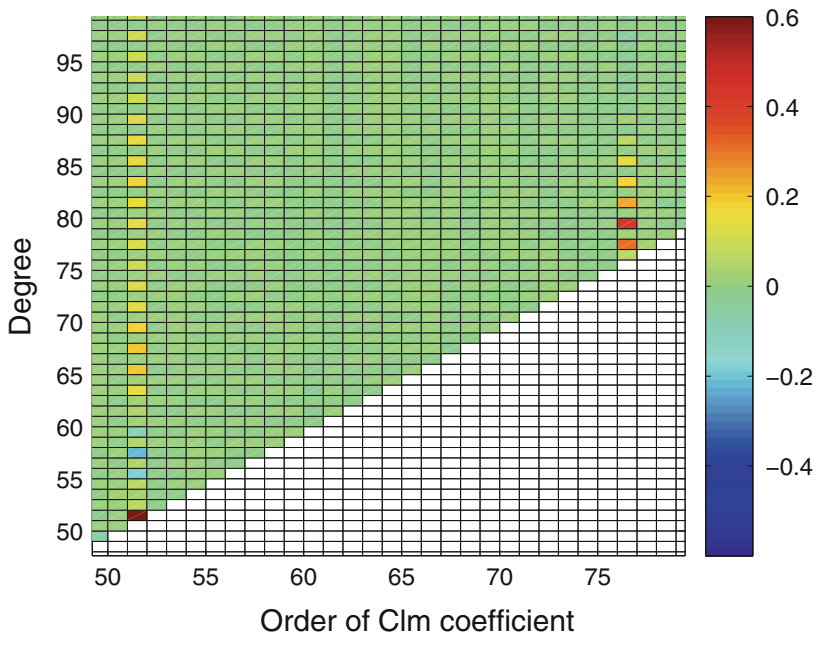

Fig. 6 Correlations with the $C(51,51)$ coefficient for an 8-day RP polar orbit

the error is calculated to be $10.38 \mathrm{~cm}$ EWH. This contradicts the expected results that the error obtained from these two cases should be more or less commensurate with each other. To explain this result, Fig. 5 presents the logarithm of the actual error in the coefficients as a function of degree and order.

Figure 5 shows that when the solution is extended to degree 100, large bands of error show up at two and three times the resonant order that do not exist in the degree 60 solutions. To study why this is the case, the covariance matrix can be analyzed. Figure 6 illustrates how the $C(51,51)$ coefficient is correlated with other coefficients and was obtained by examining the covariance matrix of a gravity solution involving only the 8-day polar RP orbit over the 13 days of the mission simulation. Note that the correlations with all coefficients are not shown in the figure, as the correlations outside of the window shown are effectively zero, as expected.

Given a spherical harmonic coefficient of a certain order, coefficients of the same order produce orbital element perturbations of identical frequency (Kaula 1966), shown by
Table 4 Dominant perturbations for $m=51$ and $m=76$ for a polar pair of satellites in an 8-day RP at $291 \mathrm{~km}$

\begin{tabular}{llll}
\hline & Period $(\mathrm{h})$ & $\Delta a(\mathrm{~cm})$ & $\Delta \omega+M\left(^{\circ}\right)$ \\
\hline$m=51$ & & & \\
$n=51,53,55, \ldots$ & 7.09 & 3.87 & $0.86 \mathrm{E}-5$ \\
$p=24,25,26, \ldots$ & & & \\
& $q=0$ & & \\
$m=76$ & & $0.67 \mathrm{E}-6$ \\
$n=77,79,81, \ldots$ & 7.09 & & \\
$p=36,37,38, \ldots$ & & & \\
$q=0$ & & & \\
\hline
\end{tabular}

the correlations at order 51 in Fig. 6. In this peculiar case, however, the period of a near resonant perturbation at order $m=51$ is identical to the period of a near-resonant perturbation at $m=76$, which also manifests itself in Fig. 6 via the correlations at $m=76$. Table 4 displays the magnitude of the largest perturbation in semimajor axis and the alongtrack direction $(\omega+M)$ for both $m=51$ and $m=76$ along with the period of the perturbation and was calculated via Rosborough and Tapley (1987).

The $p$ and $q$ variables in Table 4 are taken from Kaula's standard solution for the gravitational potential in terms of Keplerian orbital elements (Kaula 1966) while $n$ is the degree. The similarity of the perturbation frequencies leads to the filter being unable to separate them, and these bands of coefficients become poorly determined, as is reflected in Fig. 5. This error then manifests itself in the spatial plot of the recovered signals, leading to a large value of $E$. Note that similar unexpected correlations exist for orders other than the one shown here for this particular case. The above results demonstrates the importance of performing simulation studies to high degree and order.

\subsection{Selecting an inclination}

It is expected that the selected value for $i_{2}$ will have a large influence on the mission performance. Figure 7 shows the for- 

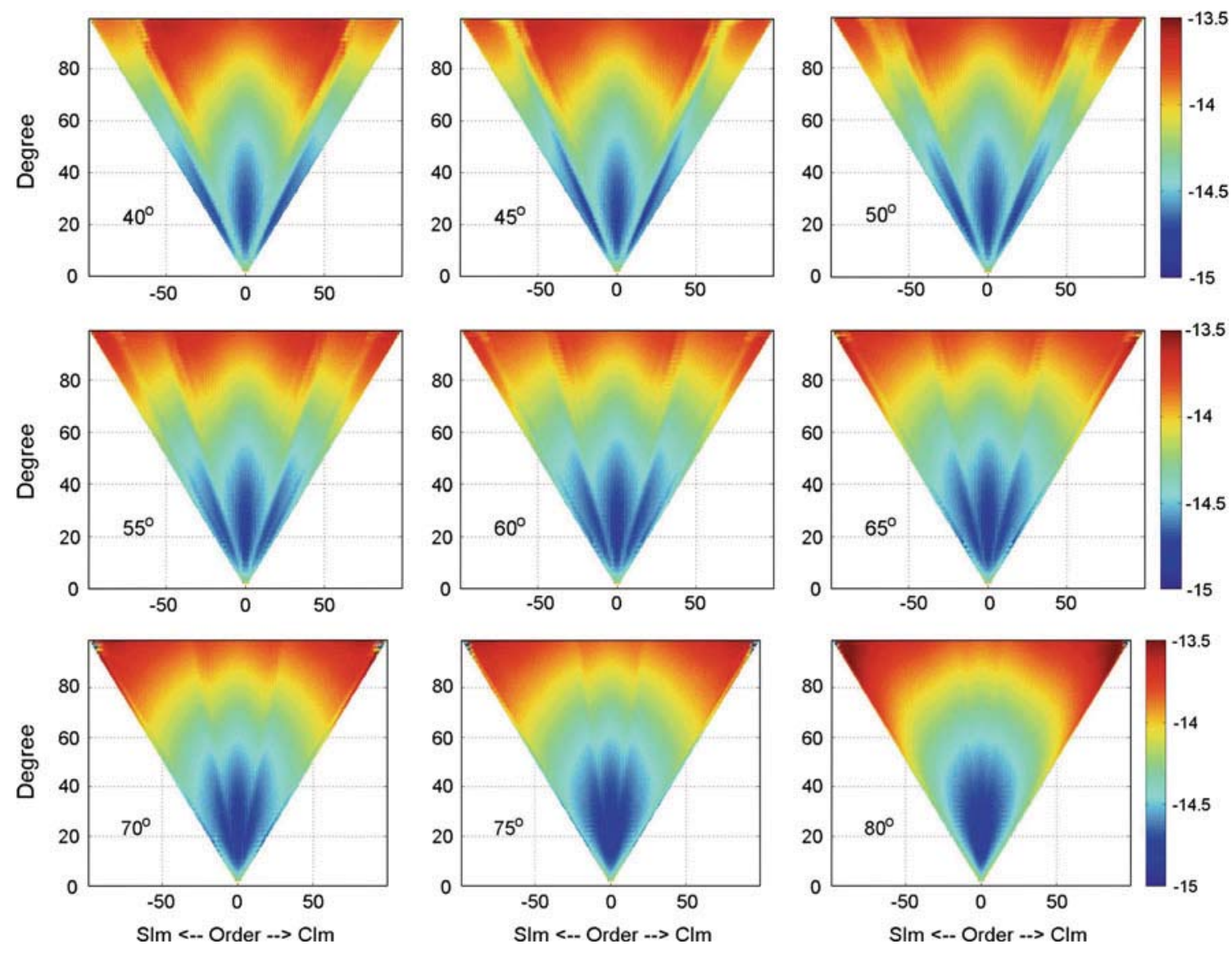

Fig. 7 Covariance analysis for a 17-day RP polar pair coupled with a 17-day RP pair of satellites at various inclinations. The logarithm of the standard deviation of the coefficients is shown

mal errors for a 17-day RP polar pair of satellites coupled with a 17-day RP pair of satellites at various inclinations. The logarithm of the formal error of each spherical harmonic coefficient is shown. Note that the difference in altitude between the satellite pairs at different inclinations will have slight influences on the formal errors. However, if one examines Fig. 3 which shows the altitude for the various inclinations of a satellite in a 17-day RP, it is seen that the difference in altitude between each of these cases is $15 \mathrm{~km}$ at a maximum. Thus, this effect should be minimal on the covariance analysis.

Figure 7 shows that for $55^{\circ} \leq i_{2} \leq 65^{\circ}$, higher degree and order tesseral harmonics are perhaps the best determined. Sectorial and near-sectorial coefficients tend to have larger errors as $i_{2}$ increases. Studying the covariance matrices alone might lead one to conclude that an inclination of approximately $60^{\circ}$ is near-optimal in the sense that the overall errors of the coefficients is lowest and the covariance matrix is fairly isotropic (no order dependence). However, geographically speaking, if the second pair of satellites flies at an inclination of $60^{\circ}$, it is seen that it provides no coverage over Greenland and does not cover a substantial amount of landmass in the northern hemisphere, including Alaska, northern Canada, northern Russia, and the Scandinavian countries. Should the second pair of satellites provide coverage over these regions, it could improve the determination of mass variations in these areas even though this is not reflected in the covariance analysis.

Therefore, it is useful to compare the error metric, $E$, as discussed in Sect. 3.2, between different inclinations. Figure 8 shows $E$ for a pair of satellites at different inclinations in a 17-day RP coupled with a polar pair of satellites in different repeat periods $(15,16$, and 17 days).

Figure 8 shows that irrespective of whether the lower inclined pair of satellites is coupled with a 15-, 16-, or 17day RP polar pair of satellites, the general trend in the error as a function of inclination is the same. Typically, the error reaches a minimum between $70^{\circ}$ and $75^{\circ}$. This is not surprising since a pair of satellites at this inclination gets fairly good coverage over the Earth while still maintaining a significant East-West component in the observable. The results from two polar pairs of satellites were not placed on this figure as the errors were so high they would distort the scale. This attests to the strength of the East-West information in 


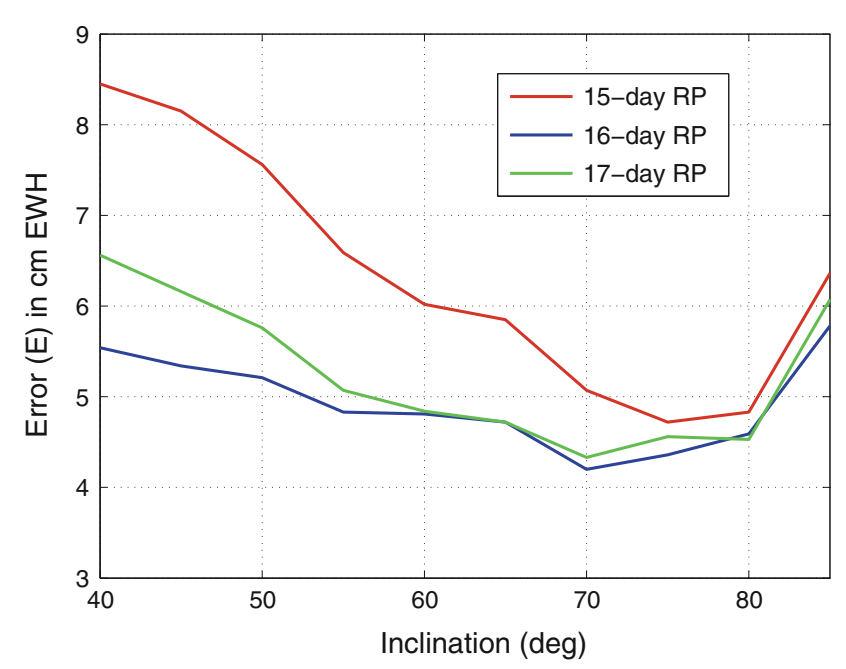

Fig. 8 Error as a function of inclination for a lower inclined pair in a 17-day RP coupled with a polar pair in 15, 16, and 17-day repeat periods

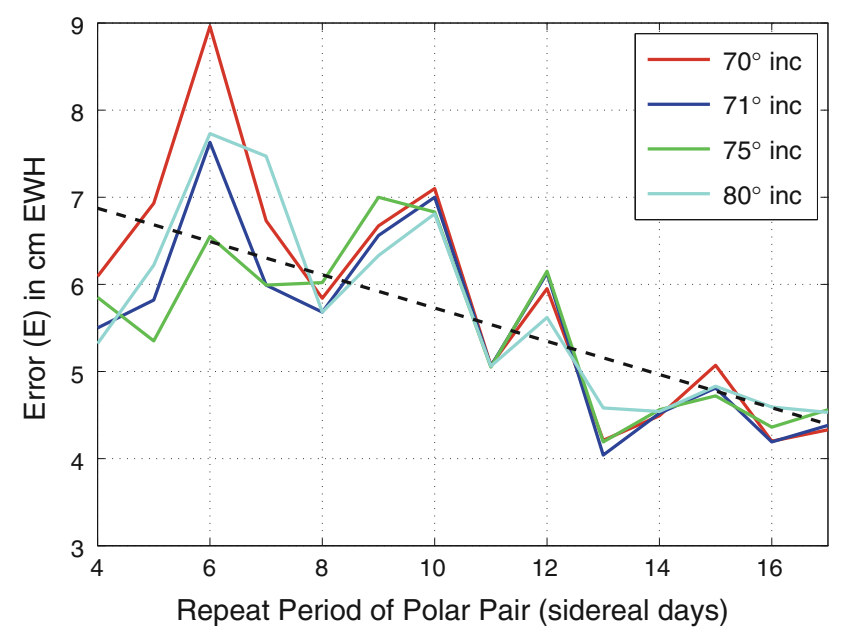

Fig. 9 Error as a function of the repeat period of a polar pair of satellites being coupled with lower inclined pair in a 17-day RP

the observable. While Fig. 8 shows an extremely small subset of the results that have been analyzed, the general trend of having the best performance for values of $i_{2}$ between $70^{\circ}$ and $75^{\circ}$ was consistent across all cases examined.

\subsection{Coupling of repeat periods}

Bender et al. (2008) suggested that having a polar orbiting pair in a shorter RP than a lower inclined pair would provide more homogeneity in the groundtrack coverage over the Earth, and thus, result in better solutions. The polar pair of satellites with a shorter RP could reduce the level of temporal aliasing errors in the polar regions as well.

Figure 9 illustrates the error as a function of different repeat periods for the polar pair of satellites coupled with a lower inclined pair of satellites $\left(70^{\circ}, 71^{\circ}, 75^{\circ}, 80^{\circ}\right)$ in a 17-day RP. Note that the range of inclinations examined in this figure is consistent with the range of inclinations that minimized the error as seen in Fig. 8.

Figure 9 shows that generally the error decreases when the lower inclined pair of satellites is coupled with a polar pair of satellites in longer repeat periods. Note that this trend exists outside of the differences in altitude between the polar pairs of satellites. In fact, plotting the error as a function of altitude of the polar pair rather than the repeat period of the polar pair does not show significant trends. It can then be assumed that the error is a stronger function of the repeat period of the polar pair of satellites than the altitude. Thus, one can assume that coupling two satellite pairs with the same repeat period will provide near-optimal results. One could argue that a global minimum may not be achieved by setting the repeat periods of the two pairs equal to each other, based on the fact that in Fig. 9 it appears that slightly smaller errors exist when the polar pair is either in a 13-day RP or a 16-day $\mathrm{RP}$ versus the 17-day RP that we have recommended. While this is true, the differences in performance between the cases is extremely small. In an effort to reduce the search space for this type of mission, we feel that invoking a $k_{1}=k_{2}$ constraint leads to near-optimal results while reducing the amount of computation time necessary to study all possible combinations of $k_{1}$ and $k_{2}$. Additional simulation results for repeat periods other than 17 days validate this statement (not shown).

\subsection{Selecting a repeat period}

After selecting a range of near-optimal inclinations for the second pair of satellites as well as enforcing the constraint that $k_{1}=k_{2}=L$, the search space for an optimal value of $L$ is substantially reduced. Table 5 shows the ten cases for which results will be displayed. Each of these cases has a lower inclined pair with an inclination around $70^{\circ}-75^{\circ}$, selected to provide the closest altitude to $290 \mathrm{~km}$. Note that the case number corresponds to the RP of the satellite pairs for convenience. It is evident that there are several repeat periods that are not shown. If a particular repeat period is not shown, for example, 12 days, this is because the altitude of one of the satellite pairs was too high for the results to be competitive with those listed. Generally speaking, due to the constraint that $k / l$ must be irreducible, repeat periods that are prime numbers have a larger range of altitudes to choose from.

Figure 10 shows the error for the cases listed in Table 5 corresponding to the polar pair of satellites with an altitude of Type I. The blue bars are the solutions obtained using the processing methodology outlined in Sect. 3.1 (referred to as 'regular' processing). Comparing these solutions, it is seen that having a repeat period in the range of 11 to 14 days provides the lowest error, with a global minimum provided by 
Table 5 Mission architectures examined to optimize the selection of a repeat period

\begin{tabular}{|c|c|c|c|c|c|c|}
\hline \multirow[t]{2}{*}{ Case } & \multicolumn{3}{|c|}{ Lower inclined pair } & \multicolumn{3}{|l|}{ Polar pair } \\
\hline & $\begin{array}{l}\text { Repeat period } \\
\text { (days) }\end{array}$ & $\begin{array}{l}\text { Inclination } \\
\left({ }^{\circ}\right)\end{array}$ & $\begin{array}{l}\text { Altitude } \\
(\mathrm{km})\end{array}$ & $\begin{array}{l}\text { Repeat period } \\
\text { (days) }\end{array}$ & $\begin{array}{l}\text { Altitude } \\
(\mathrm{km}) \text { Type I }\end{array}$ & $\begin{array}{l}\text { Altitude } \\
\text { (km) Type II }\end{array}$ \\
\hline 9 & 9 & 74 & 291 & 9 & 318 & 318 \\
\hline 11 & 11 & 70 & 300 & 11 & 306 & 332 \\
\hline 13 & 13 & 72 & 290 & 13 & 299 & 320 \\
\hline 14 & 14 & 75 & 290 & 14 & 316 & 316 \\
\hline 15 & 15 & 70 & 298 & 15 & 293 & 331 \\
\hline 17 & 17 & 71 & 290 & 17 & 305 & 322 \\
\hline 19 & 19 & 76 & 291 & 19 & 300 & 315 \\
\hline 21 & 21 & 71 & 291 & 21 & 309 & 322 \\
\hline 22 & 22 & 73 & 291 & 22 & 294 & 319 \\
\hline 23 & 23 & 75 & 291 & 23 & 292 & 317 \\
\hline
\end{tabular}

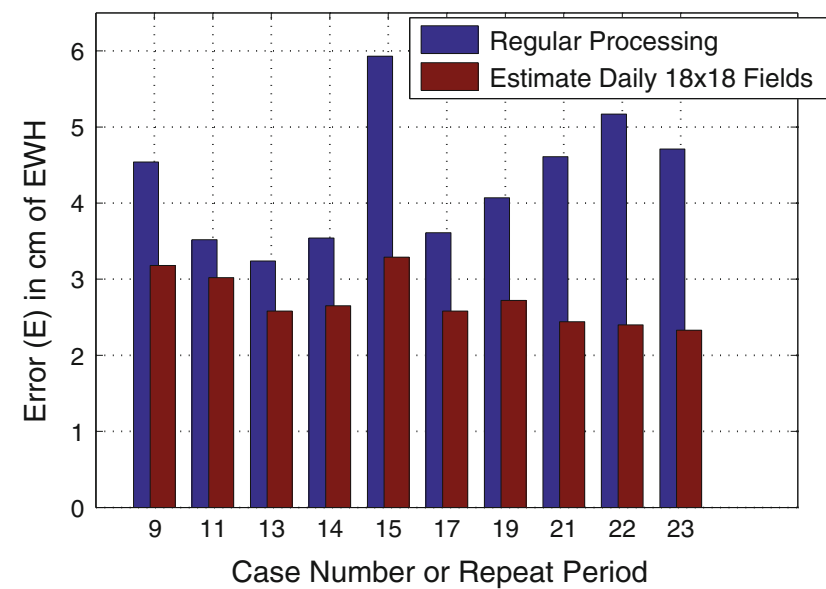

Fig. 10 Error for the cases listed in Table 5 comparing regular processing and estimating daily $18 \times 18$ gravity fields

$L=13$ days. This range of values for $L$ strikes an optimal balance between having enough data to form a good solution, but a short enough time frame where the accumulation of temporal aliasing errors is mitigated.

The red bars in Fig. 10 are obtained by invoking an alternate processing methodology where daily $18 \times 18$ gravity fields are estimated in an effort to reduce the level of temporal aliasing errors. In this process, we simultaneously estimate degrees $2-18$ each day along with degrees 19-100 over multiple days (the length of the simulation) during the inversion process. A multi-day estimate for the lower degree coefficients is then gained by simply averaging through the daily estimates. This process has been shown to reduce temporal aliasing errors from high-frequency mass variations at large spatial scales and generally results in gravity solutions with improved spatial resolution. It is described fully in Wiese et al. (2011). It is seen that estimating the daily gravity fields reduces the error substantially for all cases considered. It is also interesting to note that with this processing methodology invoked, the longer repeat periods provide the lowest errors. This makes sense, as for the case with no temporal aliasing errors the total amount of error should decrease as the square root of the number of observations. However, the reduction in errors that a 23-day RP provides over the 13-day RP case is small when one considers that 10 days of temporal resolution are sacrificed.

\subsection{Groundtrack patterns}

The three primary variables in Eq. 8 have been optimized. The last two variables, $\Delta \Omega_{12}$, and $\Delta u_{12}$ are not expected to have as large of an influence on the solution, as they only change the space-time sampling characteristics of the orbit. One thing that can be examined, however, is if certain groundtrack patterns between the two satellite pairs can be developed which will lower the errors.

In this work, it was noticed that for the case of having two polar pairs of satellites in the same RP and at the same altitude, the best solutions are obtained when $\Delta \Omega_{12}$ is set such that

$\Delta \Omega_{12}=\delta+\epsilon$,

where

$\delta=\pi\left(1+\omega_{\mathrm{e}} \sqrt{\frac{a^{3}}{\mu}}\right)$,

and

$\epsilon=\frac{2 \pi}{l}\left(\frac{1}{2}-\left(\frac{l \delta}{2 \pi}-\left\lfloor\frac{l \delta}{2 \pi}\right\rfloor\right)\right)$. 
In Eq. 12, $\delta$ shifts the relative node between the two satellite pairs exactly $180^{\circ}$ from each other plus the distance it takes for the Earth to rotate during one-half of a satellite revolution. The $\epsilon$ term is added as a correction factor such that the groundtracks of the second pair of satellites will fill in the gaps at the equator from the groundtracks of the first pair of satellites, resulting in more dense coverage. This architecture guarantees that the mutual crossing location of both satellite pairs will be at a constant low latitude $\left(\sim 7^{\circ}\right)$, rather than at the equator. This configuration appears to have substantial benefits in the case of two polar pairs of satellites, reducing the errors at the resonant orders considerably. The same magnitude of improvement is not provided when applying it to the case of a polar pair coupled with a lower inclined pair, however. The reason for this is twofold: (1) the periods of the two satellite pairs are different, and (2) the inclinations of the two pairs are different, meaning that the drift rate of the node $(\dot{\Omega})$, given in Eq. 15 (neglecting higher order terms), is different between the two cases:

$\dot{\Omega}=\frac{-3 \sqrt{\mu} J_{2} R_{\mathrm{e}}^{2} \cos i}{2\left(1-e^{2}\right)^{2} a^{\frac{7}{2}}}$.

These two differences mean that there are no consistent crossings between the two satellite pairs in either space or time. However, there are still minor improvements seen when invoking Eq. 12 to the cases in Table 5. Figure 11 shows the reduction in the level of error that this shift provides. Since $\Delta \Omega_{12}$ will not be constant for the duration of the mission due to $\dot{\Omega}$ for the lower inclined pair, the reduction in the level of errors seen in Fig. 11 represents the natural variability in the quality of the solutions due to the precession of $\Delta \Omega_{12}$.

One can now begin to think of developing a spatial groundtrack pattern for the case of having a polar pair coupled with a lower inclined pair that is consistent, as is the case when

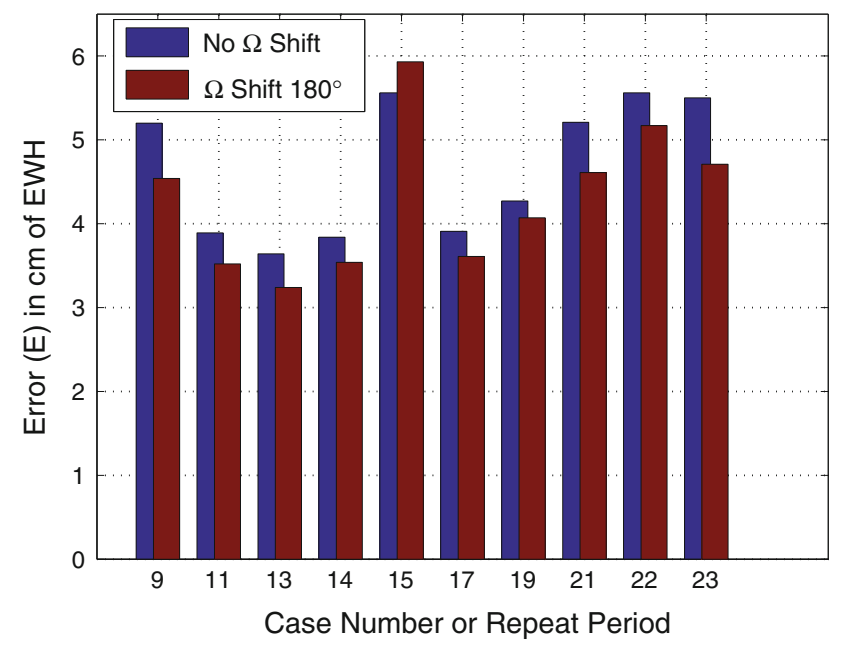

Fig. 11 Error for the cases listed in Table 5 comparing the effect of shifting the longitude of ascending node there are two polar pairs. Note that we are only interested in developing a spatial pattern $\left(\Delta \Omega_{12}\right)$, and not a temporal one $\left(\Delta u_{12}\right)$, since the difference in periods between the two satellites pairs causes a secular drift rate in this parameter $\left(\Delta \dot{u}_{12}\right)$ which cannot be controlled. One can, however, raise the altitude of the polar pair of satellites such that its period increases enough to compensate for the nodal drift rate of the lower inclined pair, thus ensuring consistent crossings at the equator in the spatial domain by solving the following equation:

$\dot{\Omega}_{\mathrm{li}} T_{\mathrm{li}}=\omega_{\mathrm{e}}\left(T_{\mathrm{pp}}-T_{\mathrm{li}}\right)$

with

$T=\dot{M}+\dot{\omega}$.

In Eq. 16 and 17, $l i$ stands for "lower inclined" and $p p$ stands for "polar pair". The period of the satellites is given by $T$. The constraint given in Eq. 16 guarantees that both pairs of satellites complete the same number of orbital revolutions in the same number of nodal days $\left(l_{1}=l_{2}\right)$ and ensures that the groundtracks of the satellites will cross each other at constant lines of latitude. Unlike the case of two polar pairs, however, the crossings will not have consistency in the time domain due to the discrepancy in periods between the two satellite pairs. Figure 12 shows the groundtrack of two pairs of satellites over South America. The groundtrack displayed in blue is from the polar pair of satellites while the groundtrack displayed in red is from the lower inclined pair of satellites. It can be seen how the two pairs always cross at the same latitude.

The modified architectures necessary to obtain the complementary groundtrack patterns described above are given by the cases involving a polar pair of satellites with an altitude of Type II in Table 5. Note that the altitude of the polar pair for each case, with the exception of Case 9 and Case 14, has been raised by approximately $20-30 \mathrm{~km}$ with respect to

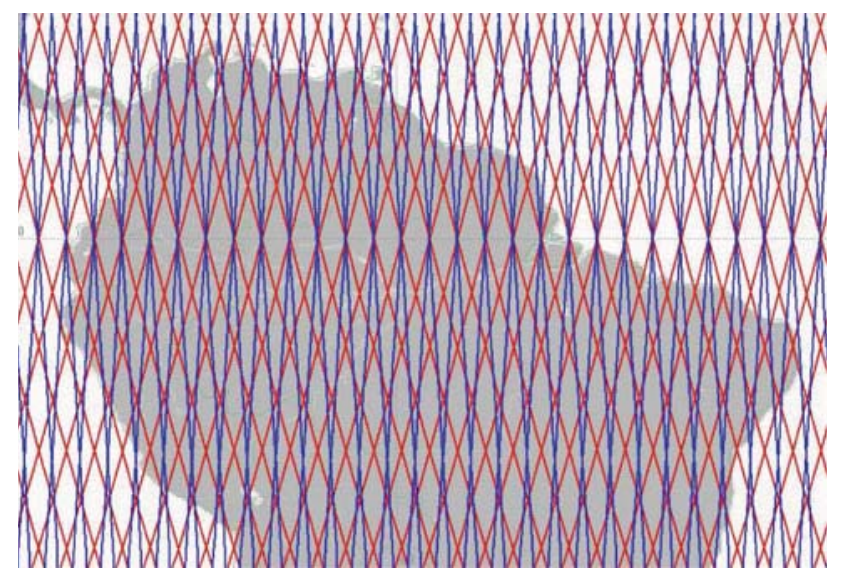

Fig. 12 Complementary groundtrack pattern shown over South America, arrived at by invoking Eq. 16 


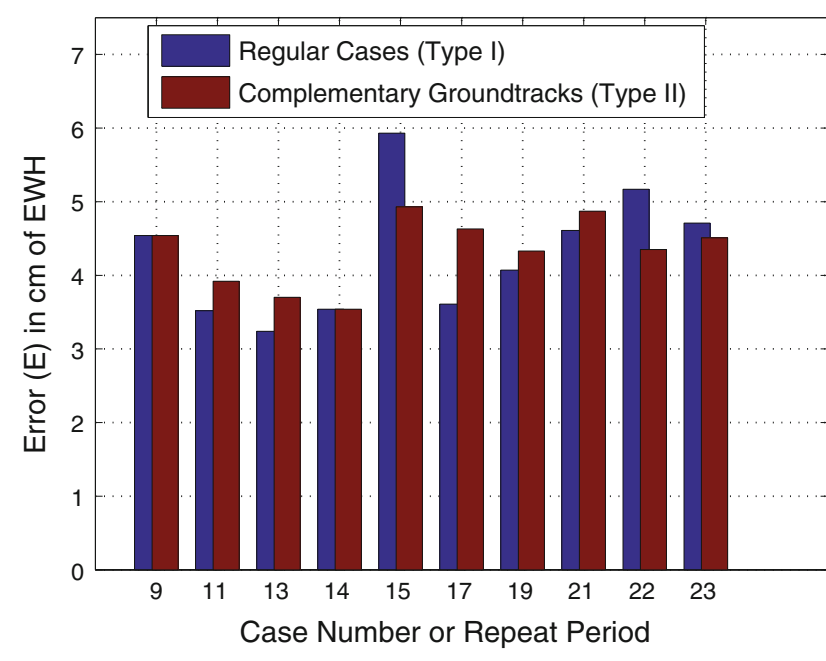

Fig. 13 A comparison of the error between the Type I and Type II architectures in Table 5

Type I. Cases 9 and 14 are the same between Type I and Type II by the fact that the polar pair selected was already at the appropriate altitude necessary for a complementary groundtrack pattern. Figure 13 compares the error from the Type I architectures in Table 5, with the lowest altitude polar pair possible, with those of Type II in Table 5, with a complementary groundtrack pattern but a slightly higher altitude for the polar pair of satellites.

Figure 13 shows a minor degradation in performance for five of the cases considered and a minor improvement in performance for three of the cases considered. Cases 9 and 14 have the same performance since they involve the same orbits. It is difficult to draw conclusions from these results. Possible benefits from flying the satellites with a complementary groundtrack pattern include the fact that the polar pair of satellites is at a higher altitude which means increased longevity due to lower atmospheric drag forces. Also, the crossings at lines of constant latitude could prove beneficial in future applications that are not yet realized, e.g., using the crossing points as constraint points for determining the geopotential at particular locations. For a gravity mapping mission, analagous to an altimeter mapping mission such as TOPEX or Envisat, there is an argument for having a geometry between the two satellite pairs in terms of the groundtracks that permits a consistent synoptic mapping of the time-variable gravity variations.

\subsection{Expected performance}

While performing an in-depth analysis of the expected scientific benefits that an optimized architecture consisting of two satellite pairs would provide over an architecture consisting of a single pair of satellites is out of the scope of this paper, a global comparison can be made using the error metrics dis- cussed in this paper. For this comparison, we select Case 13 of Type II in Table 5 consisting of a polar pair of satellites coupled with a lower inclined pair of satellites at $72^{\circ}$, both in 13-day RP orbits and possessing a complementary groundtrack pattern as discussed in Sect. 4.5. We compare this case with one polar pair of satellites in a 13-day RP at $299 \mathrm{~km}$ altitude, the performance of which is shown in Sect. 3.2. Furthermore, for the case of two satellite pairs, we co-estimate daily $18 \times 18$ gravity fields to further reduce temporal aliasing errors. As was discussed in Wiese et al. (2011), one benefit of having a polar pair coupled with a lower inclined pair of satellites is that temporal aliasing errors can be further reduced by co-estimating daily $18 \times 18$ gravity fields. Conversely, for the case of a single pair of satellites, it has been shown that while co-estimating $10 \times 10$ gravity fields every 2 days reduces temporal aliasing errors, the benefits provided by making the 2-day estimates are virtually abolished after destriping and smoothing the gravity fields. Figure 14 shows the truth signals (left), recovered signals (middle), and recovered signals after PP (right) for recovering hydrology and ice mass variations (top) and ocean bottom pressure signals (bottom) using two satellite pairs. The plots are represented out to degree 60 and are expressed in $\mathrm{cm}$ of EWH.

The PP techniques applied to these solutions are different than those applied to the single satellite pair solutions. As shown in Fig. 14, the addition of the lower inclined pair substantially reduces the level of striping in the solutions. There are certain bands of coefficients which remain correlated, however. The large errors in the high-latitude regions seen in Fig. 14 are a direct consequence of correlations in coefficients of a fixed order and same parity of degree in the range $n \geq 40$ and $3 \leq m \leq 14$. Note that these errors occur predominantly in geographical areas with latitudes higher than $72^{\circ}$, as no East-West information is present here. We found that by applying the destriping algorithm presented in Swenson and Wahr (2006) to only the selected range of spherical harmonic coefficients which have the correlated errors, we are able to remove correlated errors at high-latitude regions while leaving signals at low latitudes relatively untouched. This modified destriping algorithm has been applied to the recovered hydrology and ice signals in Fig. 14 to represent the PP solutions. The PP ocean bottom pressure signals have been destriped with this modified algorithm in addition to being smoothed with a $200-\mathrm{km}$ averaging radius. Since the ocean bottom pressure signals are smaller in magnitude than hydrology and ice signals, the signal to error ratio is substantially smaller and errors manifesting as longitudinal stripes are more apparent and hence the need to smooth the solutions.

Table 6 compares the error from one pair and two pairs for the different data reduction methodologies (whether we estimate low resolution gravity fields at a high frequency or not) along with the different PP techniques. It is seen that when no daily (or 2-day) estimates are made, and no PP is 

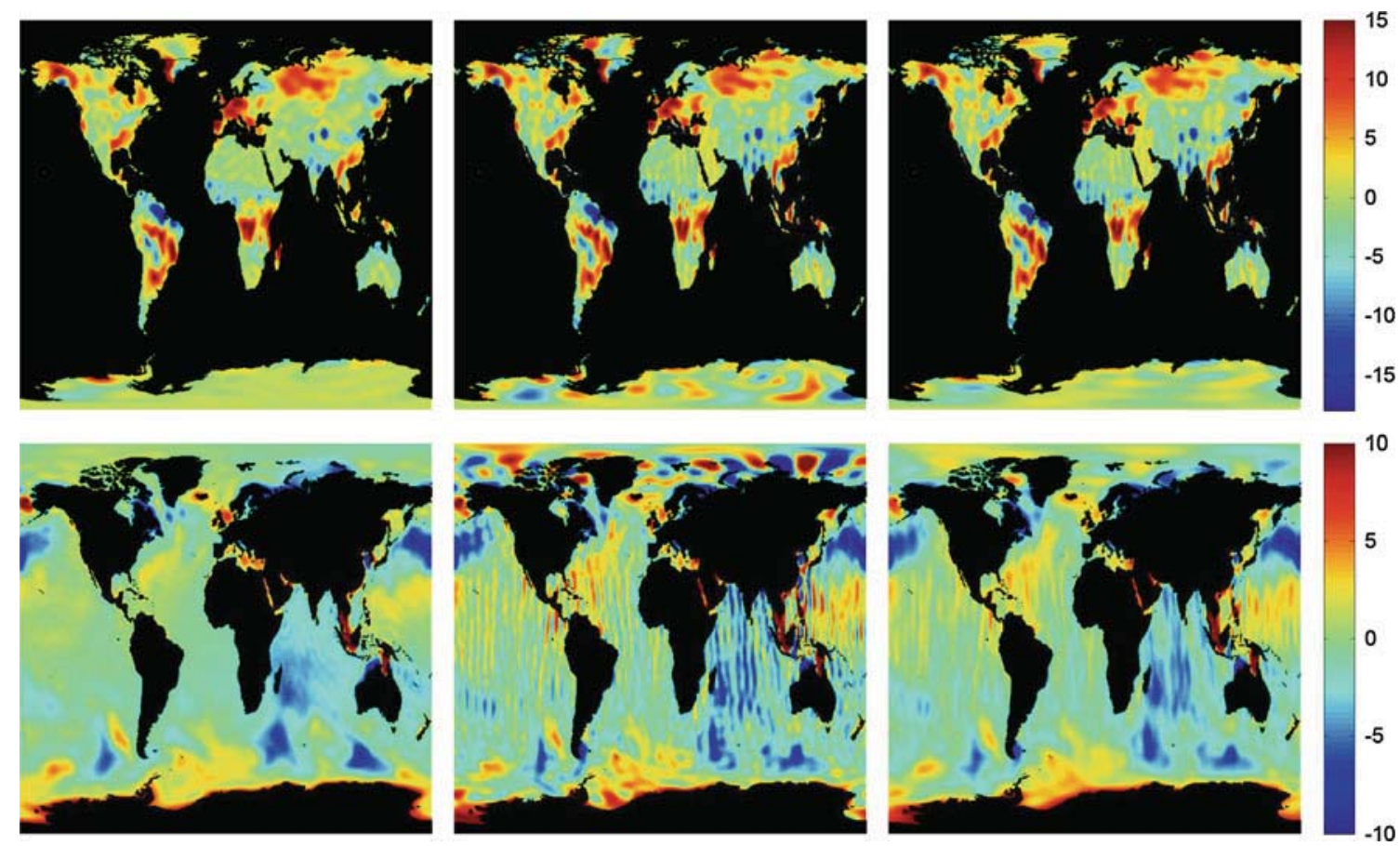

Fig. 14 Truth signals (left), recovered signals (middle), and recovered signals after PP (right) for recovering hydrology and ice mass variations (top row) and ocean bottom pressure signals (bottom row) for Case 13

of Type II in Table 5 after daily $18 \times 18$ gravity fields are co-estimated. Units are in $\mathrm{cm}$ of equivalent water height
Table 6 Signal and error comparing one pair and two pairs with various data reduction methodologies and post-processing techniques

Units are expressed in $\mathrm{cm}$ of EWH

\begin{tabular}{lllll}
\hline & Hydrology & Ice & Ocean & Average \\
\hline Signal & 4.67 & 2.12 & 2.46 & $S=3.08$ \\
Two pairs error $(\mathrm{cm})$ & & & & \\
No PP & 2.55 & 5.15 & 3.16 & $E=3.62$ \\
PP & 2.44 & 3.18 & 1.44 & $E=2.35$ \\
Est.18 $\times 18$ & 1.91 & 3.31 & 2.41 & $E=2.54$ \\
Est.18 $\times 18$, PP & 1.87 & 1.97 & 1.32 & $E=1.72$ \\
One pair error $(\mathrm{cm})$ & & & & \\
No PP & 13.97 & 7.54 & 11.04 & $E=10.85$ \\
PP & 2.47 & 2.06 & 1.71 & $E=2.08$ \\
Est.10 & 7.80 & 4.50 & 6.15 & $E=6.15$ \\
Est.10 $\times 10$, PP & 2.33 & 2.24 & 1.50 & $E=2.02$ \\
\hline
\end{tabular}

performed, the global error, $E$ is decreased by approximately $67 \%$ with the addition of the second pair of satellites. The minimal benefits of making 2 -day $10 \times 10$ gravity field estimates after PP the solutions for one pair of satellites are also seen. Table 6 illustrates that one pair of satellites actually has lower errors than two pairs of satellites if only PP is performed. The reason for this is because the PP techniques applied between the two cases are different; if the two-pair solutions are destriped and smoothed with a 300- $\mathrm{km}$ averaging radius, the error metric is actually calculated to be $E=1.99 \mathrm{~cm}$. The solutions with two satellite pairs are post-processed (PP) differently in order to preserve the information at small spatial scales that would otherwise be smoothed over. The best solutions for both cases are obtained when daily (or 2-day) estimates are made and the solutions have been PP. For this case, it is seen that two pairs of satellites offer a more modest reduction in the errors, reducing the global error by approximately $15 \%$. Having only a $15 \%$ 
reduction in error can be attributed to the performance metric used, which does a poor job of characterizing the distortions in the recovered signals from PP. For example, by destriping and smoothing (300 km radius) the truth hydrology, ice, and ocean signals, we calculate that this PP results in an error of $E=1.69 \mathrm{~cm}$. Alternately, applying the modified PP techniques for two satellite pairs to the truth signals results in an error of $E=0.53 \mathrm{~cm}$, allowing for a more accurate representation of the truth signal in the recovery. A visual comparison of Fig. 14 and Fig. 4 reveals that the solution from two pairs of satellites retains significantly more geophysical signals at small spatial scales than the post-processed one-pair solutions. Note that visually, after PP, the one-pair solutions are nearly identical whether we estimate 2 -day $10 \times 10$ gravity fields or not, with both cases smoothing through signals at small spatial scales. As such, it is anticipated that on a regional level, one will see much greater benefits with two pairs of satellites over one pair of satellites. Future work will include an in-depth analysis to quantify the performance of adding a second pair of satellites at regional levels for the various scientific areas of research.

\section{Conclusions}

Anticipating that future missions dedicated to recovering time variable gravity will use laser interferometry for intersatellite ranging, and drag-free technology for altitude control, previous studies have shown that temporal aliasing errors will dominate the error budget of the mission. One plausible way to mitigate these errors is to add an additional pair of satellites, increasing the sampling frequency of the mission, ultimately leading to a product with greater spatial resolution. Additionally, if the second pair of satellites is at a lower inclination, the East-West sensitivity of the observable is improved, decreasing the longitudinal striping in the solutions. The goal of this paper was to optimize the orbits of two satellites pairs to provide increased spatial resolution in determining hydrology, ice mass variations, and ocean bottom pressure signals globally.

While the search space for such a problem is, by nature, infinite, numerical simulations to degree and order 100 were implemented in an effort to reduce it. A search space originally consisting of 15 variables was reduced to two variables with primary impact on mission performance: the inclination of one of the satellite pairs (the other pair is assumed to be polar), and the repeat periods of both pairs of satellites (shown to be near-optimal when they are equal to each other). In this study we considered only circular orbits in repeating groundtracks, a minimum allowable altitude of $290 \mathrm{~km}$ based on a projected 10-year mission lifetime, and assumed a 100$\mathrm{km}$ inter-satellite separation distance between each pair of satellites. It was found that an optimal value for the inclina- tion of the second pair of satellites is between $70^{\circ}$ and $75^{\circ}$, while an appropriate range for the repeat periods of both satellite pairs is between 11 and 14 days. The absolute lowest errors are given when both satellite pairs are in a 13-day repeat period, one being polar at an altitude of $299 \mathrm{~km}$, and the other inclined at $72^{\circ}$ at an altitude of $290 \mathrm{~km}$. It should be noted that the results of this study are influenced by the targeted altitude for the mission as well as the scientific goals of the mission.

The notion of optimizing the relative change in node and the argument of latitude between the two pairs was discussed in relation to creating complementary groundtrack patterns. It was shown that by raising the altitude of the polar pair, the nodal drift rate of the lower inclined pair can be compensated for such that a groundtrack pattern with crossings at constant lines of latitude is created. While numerical simulation results imposing this constraint were not conclusive as to whether this definitively results in improved mission performance, there is an argument for having a geometry that permits consistent global mapping of the gravity field. Finally, the importance of extending simulations to high degree and order was shown.

Results showed that with an optimized architecture consisting of two satellite pairs, global errors are reduced by $67 \%$ (no post-processing) with the addition of the second pair of satellites over one pair of satellites. After each set of solutions has been destriped and smoothed with appropriate methods, and daily (or 2-day) gravity fields are estimated, it was seen that two pairs of satellites offered a more modest reduction in error of $15 \%$ over one pair of satellites. Visually, however, it was seen that the two-pair solution retains significant geophysical signals at small spatial scales which are smoothed and damped in the one-pair solutions. Future work will involve an in-depth examination of the expected scientific benefits of an optimized two-pair architecture, extending the analysis to local regions as well as longer time spans.

Acknowledgments This research was funded by the National Science Foundation Graduate Fellowship Program, the National Defense Science and Engineering Graduate Fellowship Program, and the NASA GRACE Science Team investigation (NNX08AH63G). The authors would like to thank NASA Goddard Space Flight Center for providing GEODYN and SOLVE to perform the numerical simulations, along with the NCEP model. We acknowledge Jean-Paul Boy (EOST/University of Strasbourg, France) for providing the ECMWF-derived and MOG2D-derived datasets in this study to GSFC. Additionally, we thank ESA and the Institute of Astronomical and Physical Geodesy (IAPG) at the Technical University of Munich for providing the ice model used in the simulations.

\section{References}

Bender PL, Hall JL, Ye J, Klipstein WM (2003) Satellite-satellite laser links for future gravity missions. Space Sci Rev 108:377-384

Bender PL, Wiese DN, Nerem RS (2008) A possible dual-GRACE mission with 90 degree and 63 degree inclination orbits. In: Pro- 
ceedings of the third international symposium on formation flying, missions and technologies. ESA/ESTEC, Noordwijk, pp 1-6

Bruinsma S, Lemoine JM, Biancale R, Valès N (2010) CNES/GRGS 10-day gravity field models (release 2) and their evaluation. Adv Space Res 45:587-601

Carrère L, Lyard F (2003) Modeling the barotropic response of the global ocean to atmospheric wind and pressure forcing. Geophys Res Lett 30(6):1275. doi:10.1029/2002GL016473

Center for Space Research (2011) GRACE orbital configuration. http:// www.csr.utexas.edu/grace/operations/configuration.html

Colombo O (1984) The global mapping of gravity with two satellites. Tech. Rep. 3, Netherlands, Geodetic Commission, Publications on Geodesy, New Series

Drinkwater M, Haagmans R, Muzzi D, Popescu A, Floberghagen R, Kern M, Fehringer M (2007) The GOCE gravity mission: ESA's first core explorer. In: Proceedings of the Third GOCE User Workshop. Frascati, Italy, ESA SP-627, pp 1-7

Elsaka B (2010) Simulated satellite formation flights for detecting temporal variations of the earth's gravity field. $\mathrm{PhD}$ thesis, University of Bonn

Fehringer M, Floberghagen R, Muzi D, Steiger C, Pineiro J (2010) Abstract G33B-01 presented at 2010 Fall Meeting AGU. San Francisco

Flechtner F (2007) AOD1B product description document for product releases 01 to 04 . University of Texas at Austin, GRACE 327-750, CSR Publ. GR-GFZ-AOD-0001 Rev. 3.1, 43 p

Förste C, Schmidt R, Stubenvoll R, Flechtner F, Meyer U, König R, Neumayer H, Biancale R, Lemoine J, Bruinsma S, Loyer S, Barthelmes F, Esselborn S (2008) The GFZ/GRGS satellite and combined gravity field models EIGEN-GL04S1 and EIGEN-GL04C. J Geodesy 82(6):331-346. doi:10.1007/s00190-007-0183-8

Han SC, Ditmar P (2008) Localized spectral analysis of global satellite gravity fields for recovering time-variable mass redistributions. $\mathrm{J}$ Geodesy 82:423-430. doi:10.1007/s00190-007-0194-5

Han SC, Simons FJ (2008) Spatiospectral localization of global geopotential fields from the gravity recovery and climate experiment (GRACE) reveals the coseismic gravity change owing to the 2004 Sumatra-Andaman earthquake. J Geophys Res 113:B01405. doi:10.1029/2007JB004927

Kalnay E, Kanamitsu M, Kistler R, Collins W, Deaven D, Gandin L, Iredell M, Saha S, White G, Woollen J, Zhu Y, Chelliah M, Ebisuzaki W, Higgins W, Janowiak J, Mo KC, Ropelewski C, Wang J, Leetmaa A, Reynolds R, Jenne R, Joseph D (1996) The NCEP/NCAR 40-year reanalysis project. Bull Am Meteorol Soc 77:437-470

Kaula WM (1966) Theory of Satellite Geodesy. Blaisdell Publishing Company, Waltham

Klinker E, Rabier F, Kelly G, Mahfouf JF (2000) The ECMWF operational implementation of four-dimensional variational assimilation. III: Experimental results and diagnostics with operational configuration. Q J R Meteorol Soc 126(564):1191-1215

Klokočník J, Wagner CA, Kostelecký J, Bezděk A, Novák P, McAdoo D (2008) Variations in the accuracy of gravity recovery due to ground track variability: GRACE, CHAMP, and GOCE. J Geodesy 82:917-927

Loomis B (2009) Simulation study of a follow-on gravity mission to GRACE, PhD thesis, University of Colorado, Boulder

Lyard F, Lefèvre F, Letellier T, Francis O (2006) Modelling the global ocean tides: modern insights from FES2004. Ocean Dynamics 56:394-415. doi:10.1007/s10236-006-0086- $\mathrm{x}$

Marchetti P, Blandino JJ, Demetriou MA (2008) Electric propulsion and controller design for drag-free spacecraft operation. J Spacecraft Rockets 45(6):1303-1315. doi:10.2514/1.36307

Mueller G, Thorpe I, Mcnamara P, Camp J (2005) Laser frequency stabilization for LISA. NASA/TM-2005-212794, NASA Goddard Space Flight Center
Pavlis DE, Deng C, McCarthy JJ (2010) GEODYN operations manual. Contractor report, SGT, Inc., Greenbelt

Pierce R, Leitch J, Stephens M, Bender P, Nerem R (2008) Intersatellite range monitoring using optical interferometry. Appl Opt 47:50075019

Ray RD (1999) A global ocean tide model from TOPEX/POSEIDON altimetry: GOT99.2. NASA technical memorandum 209478, Goddard Space Flight Center

Rodell M, House P, Jambor U, Gottschalck J, Mitchell K, Meng CJ, Arsenault K, Cosgrove B, Radakovich J, Bosilovich M, Entin JK, Walker JP, Lohmann D, Toll D (2004) The global land data assimilation system. Bull Am Meteorol Soc 85:381-394. doi:10.1175/ BAMS-85-3-381

Rosborough GW, Tapley BD (1987) Radial, transverse, and normal satellite position perturbations due to the geopotential. Celestial Mech 40:409-421

Rowlands DD, Ray RD, Chinn DS, Lemoine FG (2002) Short-arc analysis of intersatellite tracking data in a gravity mapping mission. $\mathrm{J}$ Geodesy 76:307-316. doi:10.1007/s00190-002-0255-8

Simons FJ, Dahlen FA, Wieczorek MA (2006) Spatiospectral concentration on a sphere. SIAM Rev 48:504-536. doi:10.1137/ S0036144504445765

St Rock B, Blandino JJ, Demetriou MA (2006) Propulsion requirements for drag-free operation of spacecraft in Low Earth Orbit. J Spacecraft Rockets 43(3):594-606. doi:10.2514/1.15819

Swenson S, Wahr J (2002) Methods for inferring regional surfacemass anomalies from gravity recovery and climate experiment (GRACE) measurements of time-variable gravity. J Geophys Res 107(B9):2193. doi:10.1029/2001JB000576

Swenson S, Wahr J (2006) Post-processing removal of correlated errors in GRACE data. Geophys Res Lett 33:L08402. doi:10.1029/ 2005GL025285

Tapley BD, Bettadpur S, Ries JC, Thompson PF, Watkins MM (2004a) Grace measurements of mass variability in the earth system. Science 305:503-505. doi:10.1126/science.1099192

Tapley BD, Bettadpur S, Watkins M, Reigber C (2004b) The gravity recovery and climate experiment: mission overview and early results. Geophys Res Lett 31:L09607. doi:10.1029/ 2004GL019920

Ullman R (1997) SOLVE program mathematical formulation. Rep. HSTX- G and G-9201

Vallado DA (2001) Fundamentals of astrodynamics and applications: second edition. Microcosm Press and Kluwer Academic Publishers, El Segundo

van Dam T, Visser P, Sneeuw N, Losch M, Gruber T, Bamber J, Bierkens M, King M, Smit M (2008) Monitoring and modelling individual sources of mass distributions and transport in the Earth system by means of satellites. Tech. rep., Final Report, ESA Contract 20403

Visser PNAM, Sneeuw N, Reubelt T, Losch M, van Dam T (2010) Space-borne gravimetric satellite constellations and ocean tides: aliasing effects. Geophys J Int 181(2):789-805. doi:10.1111/j. 1365-246X.2010.04557.x

Visser PNAM, Schrama EJO, Sneeuw N, Weigelt M (2011) Dependency of resolvable gravitational spatial resolution on space-borne observation techniques. In: Kenyon S, Pacino MC, Marti U (eds) Proceedings of the 2009 IAG Symposium, Buenos Aires, Argentina, 31 August - 4 September 2009. International Association of Geodesy Symposia, vol. 136. Springer, Heidelberg. ISBN 978-3-64220337-4

Wagner C, McAdoo D, Klokočník J, Kostelecký J (2006) Degradation of geopotential recovery from short repeat-cycle orbits: application to GRACE monthly fields. J Geodesy 80:94-103. doi:10.1007/ s00190-006-0036-X

Wahr J, Molenaar M (1998) Time variability of the Earth's gravity field: hydrological and oceanic effects and their possible detection using GRACE. J Geophys Res 103(B12):30205-30229 
Wieczorek MA, Simons FJ (2005) Localized spectral analysis on the sphere. Geophys J Int 162:655-675. doi:10.1111/j.1365-246X. 2005.02687.x

Wiese DN, Folkner WM, Nerem RS (2009) Alternative mission architectures for a gravity recovery satellite mission. J Geodesy 83:569_ 581. doi:10.1007/s00190-008-0274-1
Wiese DN, Visser PNAM, Nerem RS (2011) Estimating low resolution gravity fields at short time intervals to reduce temporal aliasing errors. Adv Space Res. doi:10.1016/j.asr.2011.05.027

Young BC, Cruz FC, Itano WM, Bergquist JC (1999) Visible lasers with subhertz linewidths. Phys Rev Lett 82(19):3799-3802 